\title{
Ecografía de bolsillo en Medicina Interna
}

\author{
Pocket Mobile Echography in Internal Medicine
}

\author{
G. López LLoret ${ }^{1,2}$, G. López Martínez ${ }^{1,2}$, S. López Palmero ${ }^{1,2}$, F. Diez García ${ }^{1,2}$ \\ ${ }^{1}$ Servicio de Medicina Interna. Complejo Hospitalario Torrecárdenas. Almería. ${ }^{2}$ Grupo de Ecografía Clínica, SEMI
}

\section{Resumen}

La utilización de dispositivos de ecografía de bolsillo a la cabecera del paciente, por el propio internista, va a redefinir probablemente la exploración física y la aproximación al diagnóstico en Medicina Interna. La comercialización reciente de ecógrafos portátiles, que pueden transportarse en el bolsillo de la bata, permite la realización de ecografías a pie de cama y profundizar en los hallazgos obtenidos con la historia clínica y la exploración física en tiempo real, mejorando la toma de decisiones a la cabecera del paciente. Además proporciona una ayuda importante en la realización de determinadas pruebas diagnósticas como toracocentesis y paracentesis. El desarrollo e implantación de esta tecnología puede revolucionar la aproximación médico-paciente en los próximos años.

\section{Introducción}

El desarrollo de nuevas tecnologías en la miniaturización e informática portátil y su aplicación en el diagnóstico y terapéutica médica, marcarán probablemente el ritmo de la Medicina del siglo XXI y revolucionarán el acto básico de atención médico-paciente, aportando instrumentos de precisión que permitirán obtener información mucho más allá de la obtenida con la historia y la exploración clínica. Los nuevos ecógrafos tamaño "de bolsillo" (Figura 1) permiten disponer de una tecnología de alta precisión "en el instante" y "en cualquier lugar asistencial". Probablemente, el examen físico del futuro contará con la ecocardiografía como parte de la evaluación ${ }^{1}$ y los nuevos dispositivos de ecografía de bolsillo están llamados a convertirse en el fonendo del futu$r^{2,3}$. Sin embargo aún no se conoce bien cuál puede ser la utilidad real de esta nueva tecnología en distintos lugares asistenciales y en manos de distintos profesionales sanitarios. La evolución y difusión de los dispositivos de telefonía móvil y los llamados Smartphone (teléfonos inteligentes) de última generación con la opción de intercambio de imágenes a distancia y la posibilidad de incorporar adaptadores de sondas ecográficas en el mismo dispositivo abren un interesante camino en la interpretación remota de resultados ${ }^{4}$. La utilización de los ultrasonidos no es exclusiva de radiólogos y cardiólogos, pueden ser realizadas e interpretadas por otros médicos con la formación suficiente ${ }^{5}$. La utilidad y la seguridad de la ecografía a pie de cama realizada por los clínicos se ha demostrado plenamente en los Servicios de Urgencias y las Unidades de Cuidados Intensivos (UCI) $)^{6-9}$. También su viabilidad y fiabilidad ${ }^{10}$. En los Servicios de Medicina Interna, algunos autores defienden que su utilización aportará

\begin{abstract}
The use of pocket ultrasound devices at the patient's bedside by the internist will probably redefine physical examination and diagnostic approach in Internal Medicine. The recent marketing of these portable ultrasound devices, which may be carried by the doctor in his coat pockett, allows performing bedside ultrasound explorations, and to expand and detail the findings collected with the clinical history and the physical examination in real time, improving decision making at the bedside. It also provides an important aid in performing certain diagnostic tests such as thoracentesis and paracentesis. The development and implementation of this technology can revolutionize the doctor-patient approach in the coming years.
\end{abstract}

ventajas a los pacientes, mejorando el proceso y tiempo de diagnóstico y el acceso rápido al tratamiento adecuado, reduciendo el tiempo de hospitalización ${ }^{11}$.

Figura 1. Ecógrafo de bolsillo: toracocentesis guiada por ecografía

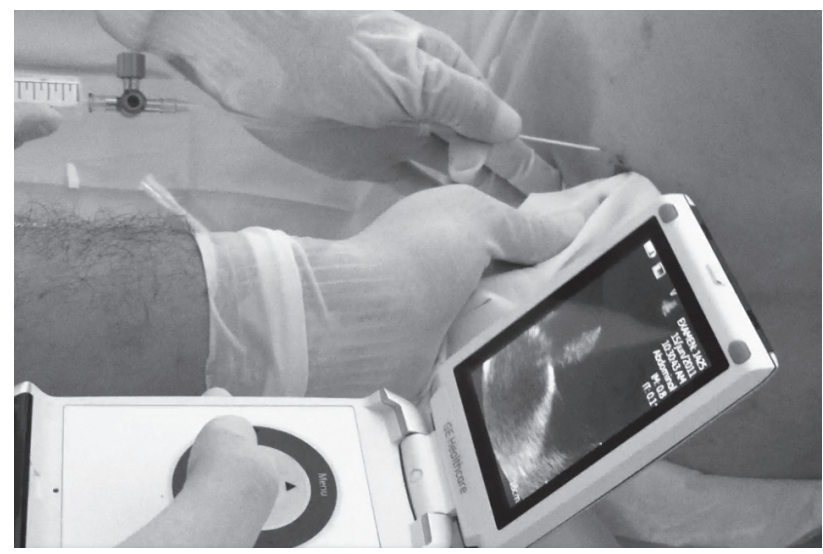

La utilización de dispositivos de ecografía de bolsillo por el clínico (fonendo del Siglo XXI), creemos que aportará importantes ventajas a los pacientes, podrá reducir el número de exploraciones de imagen de rutina, y probablemente permita seleccionar las exploraciones de imagen necesarias (Ecocardiograma, TAC, RMN), de una forma más eficiente. Desde Junio de 2010 comenzamos a utilizar un dispositivo de ecografía de bolsillo con doppler color en nuestro Servicio de Medicina Interna. Desde entonces cuatro internistas han recibido adiestramiento en ecografía clínica y se han realizado diversos cursos básicos de formación dirigidos a médicos adjuntos y residentes. Se ha impulsado también el aprendizaje de los alumnos de medicina en sus prácticas por el área clínica. Nuestro proyecto se aceptó como idea 
Figura 2. Ecógrafo en bolsillo

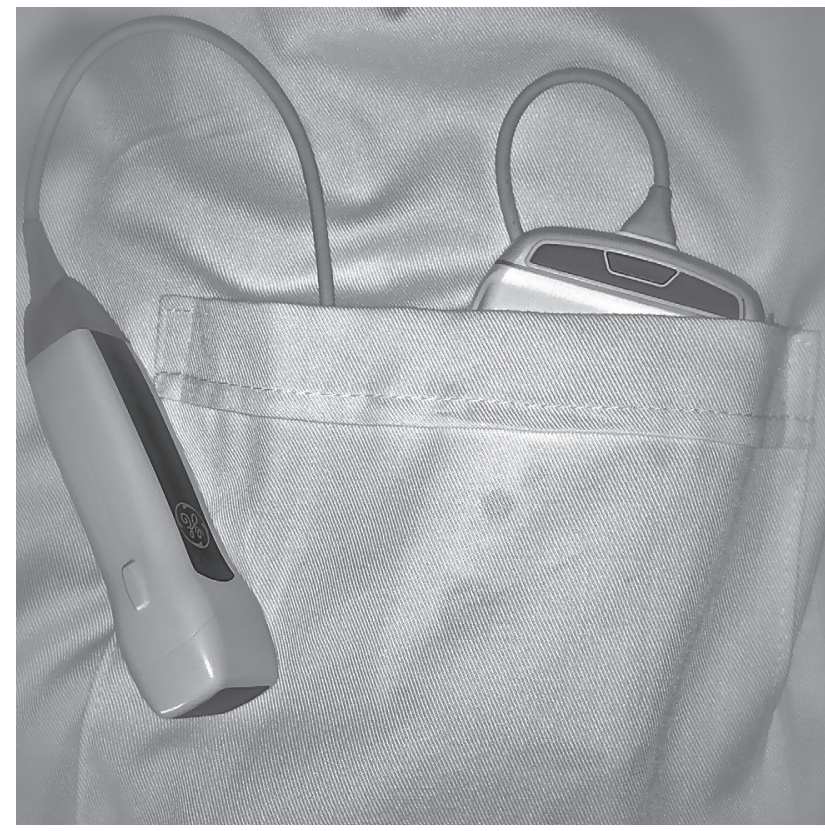

y práctica innovadora en el Banco de Prácticas Innovadoras de la Junta de Andalucía (www.saludinnova.com). El objetivo fundamental fue avanzar en el conocimiento de la utilidad de la ecografía de bolsillo en un Servicio de Medicina Interna y comprobar la concordancia de los hallazgos obtenidos con el dispositivo portátil con los obtenidos con las ecografías convencionales. Nuestro estudio se ha centrado en ecografía cardiaca, torácica y abdominal fundamentalmente.

Junto a la práctica clínica y la utilización diagnóstica de los equipos, se ha realizado de forma simultánea una labor de difusión de imágenes obtenidas con el ecógrafo de bolsillo, en forma de vídeos a través de la web, creando un canal en youtube y una página web con interés docente (www. ecografiadebolsillo.com). La recogida de datos, evolución y valoración de resultados preliminares se ha difundido en distintos Congresos (Congreso de Enfermedades Autoinmunes, Jaén 2011, Congreso Andaluz de Cardiología, Sevilla 2011, Congreso de la Sociedad Andaluza de Medicina Interna, Granada 2011, Congreso de Neurología, Barcelona 2011, Congreso de Calidad, Almería 2011) y se mantienen actualmente abiertas varias líneas de investigación. Asimismo hemos colaborado desde el principio con el grupo de Ecografía Clínica de la SEMI, su página web, y el grupo de Facebook. En las líneas siguientes queremos compartir nuestra experiencia y los resultados que disponemos hasta el momento en forma de estudio de concordancia.

\section{Información técnica}

En la realización de las ecografías se han utilizado tres dispositivos portátiles con Doppler color, VSCAN de General Electric (Figura 1). Las principales características de estos ecógrafos de bolsillo son las siguientes: el dispositivo consta de una unidad que incluye la pantalla y las teclas

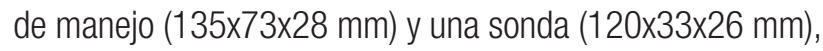
con un peso total de $390 \mathrm{gr}$, permitiendo su transporte en el bolsillo de una bata (Figura 2). La pantalla, orientada en vertical (3:4), de $8,9 \mathrm{~cm}$ (diagonal) tiene una resolución de $240 \times 320$ pixels. El ajuste de ganancia es automático para tres programas definidos (cardiaco, abdominal, ginecológico). La imagen se visualiza en escala de grises y en modo doppler color, pudiendo ajustarse con las teclas del cursor el brillo, profundidad y posición del doppler color. El ancho de banda de la sonda es 1,7 a 3,8 MHz. El aparato posee función automática de detección de ciclo cardiaco. Con el VSCAN se pueden medir distancias durante la exploración. La valoración de áreas y la posterior estimación de la FE se realizó con imágenes fijas previamente grabadas y vídeos de corta duración, empleándose el software VSCAN Gateway, que permite la edición y el archivo digital (en formato MP4). El aparato dispone de una sonda tipo sectorial y carece de modo $M$.

Se ha utilizado el programa predefinido como cardiaco para la ecocardiografía y el abdominal para valoración de abdomen y para la diferenciación de derrame pleural y/o atelectasia (Figura 3). Para la valoración de grandes vasos (aorta y cava) se han utilizado ambos. Las imágenes, tanto fijas como en vídeo (secuencias de corta duración), se han archivado utilizando el programa de software que acompaña al

Figura 3. Derrame pleural

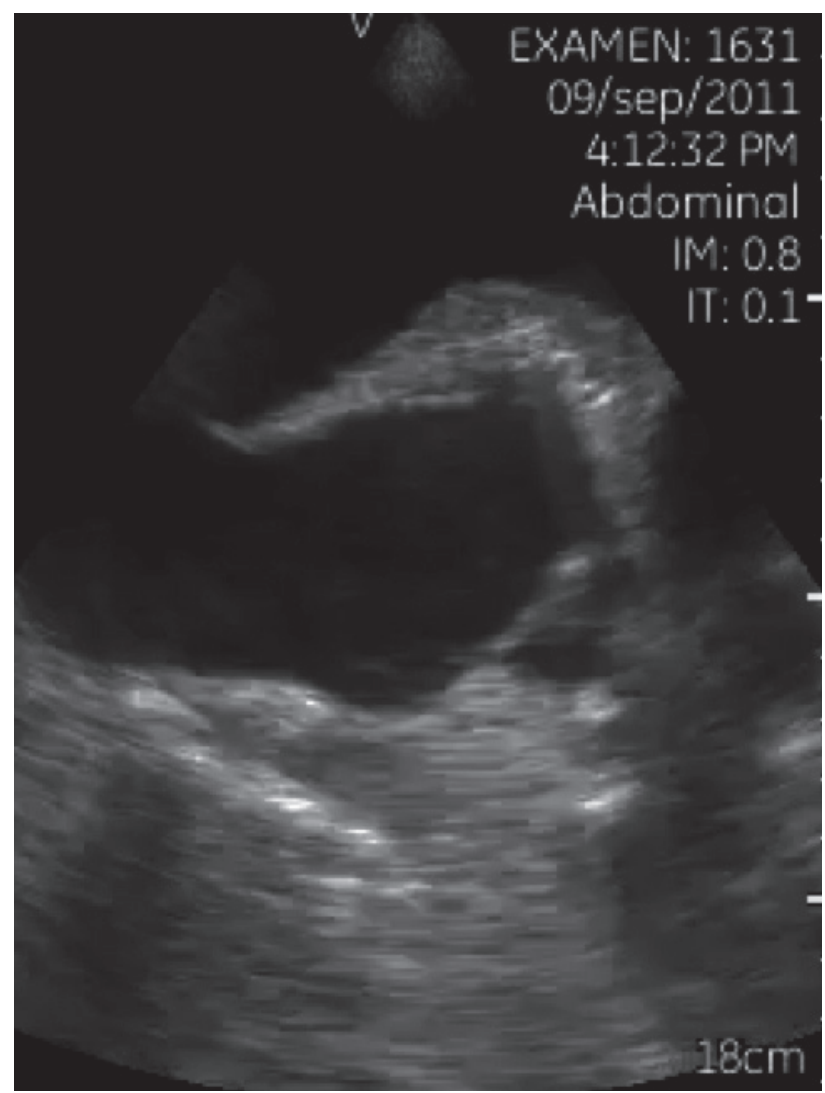


Figura 4. Ascitis

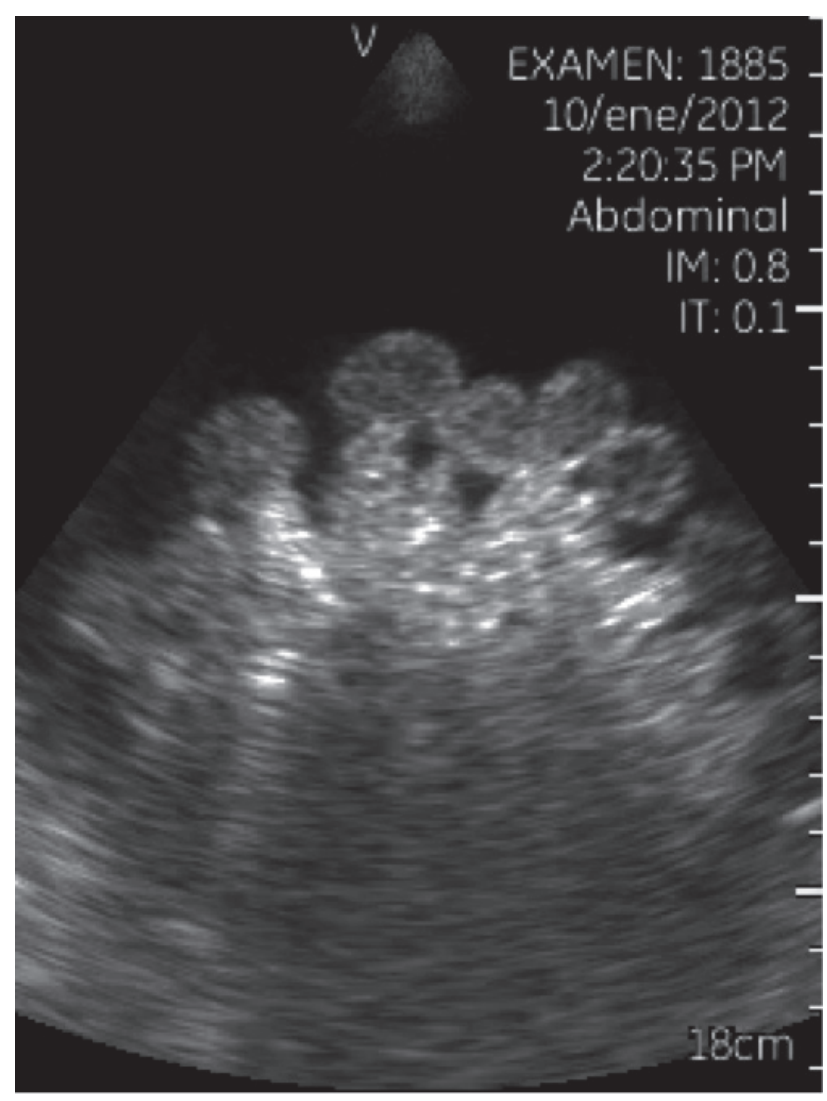

dispositivo para PC (VSCAN Gateway). La edición de vídeo se realizó con Moviemaker (PC), Imovie (Mac) y Adobe Premiere 6 para Mac. Se ha utilizado gel de ecografía convencional aportado por el Servicio de Farmacia Hospitalaria.

\section{Selección y descripción de participantes}

Las ecografías se han realizado fundamentalmente en pacientes hospitalizados en Medicina Interna, área de Consultas Externas y Urgencias, de cualquier edad, sexo y raza, en cualquier horario, incluyendo periodos de jornada laboral y guardias, a la cabecera del paciente, en su propia cama. También se ha colaborado con otros Servicios Médicos y Quirúrgicos de nuestro Hospital, como Neurología, Neumología, Digestivo, Nefrología, Cardiología, UVI, Traumatología y Neurocirugía, y por tanto, se han incluido pacientes ingresados en dichos Servicios.

Las ecografías se realizaron durante la exploración física del paciente 0 inmediatamente después, en particular en aquellos pacientes en los que se detectó algún signo guía como: soplo cardiaco, arritmia, cardiomegalia radiológica, disnea de origen incierto, HTA, hipoventilación o asimetrías en la auscultación del tórax, distensión abdominal, sospecha de ascitis, globo vesical, oligo-anuria, ictericia, signos de insuficiencia cardiaca, palpación de visceromegalias o masa abdominal. Además se ha empleado el ecógrafo de bolsillo para la detección de líquido pleural ó ascítico (Figuras 3 y 4) y en la ayuda a la loca- lización del punto de punción para toracocentesis y paracentesis (Figura 1). De forma preferente utilizamos el dispositivo de bolsillo en aquellos pacientes con movilidad reducida y en ancianos con mayor dificultad para el traslado a las Unidades de Radiodiagnóstico y de Ecocardiografía.

En la mayoría de los casos pudimos comparar los resultados obtenidos con el ecógrafo de bolsillo con las técnicas de imagen convencionales (ecocardiograma, ecografía abdominal, TAC), realizadas en las Unidades correspondientes, permitiendo analizar así la concordancia de los resultados obtenidos con ambas técnicas.

Al tratarse de un procedimiento inocuo, no se plantearon contraindicaciones a su uso ni criterios de exclusión salvo la negativa de pacientes o familiares. Se desestimaron aquellas exploraciones que carecían de la calidad mínima necesaria debido a "mala ventana acústica" fundamentalmente por interposición de gas en abdomen con mala transmisión ultrasónica, o la carencia de visualización correcta de al menos dos planos en los cortes de ecocardiografía. Se informó oportunamente del procedimiento a los pacientes, y se ha guardado en todo momento la confidencialidad y el derecho a la intimidad. Finalmente, se recogieron resultados en relación a la satisfacción del usuario como control de calidad.

Los resultados se analizaron con el programa SPSS versión 19. Se utilizó el Índice Kappa (porcentaje de acuerdo por encima del esperado por el azar) como test de concordancia.

Figura 5. Mixoma de aurícula izquierda

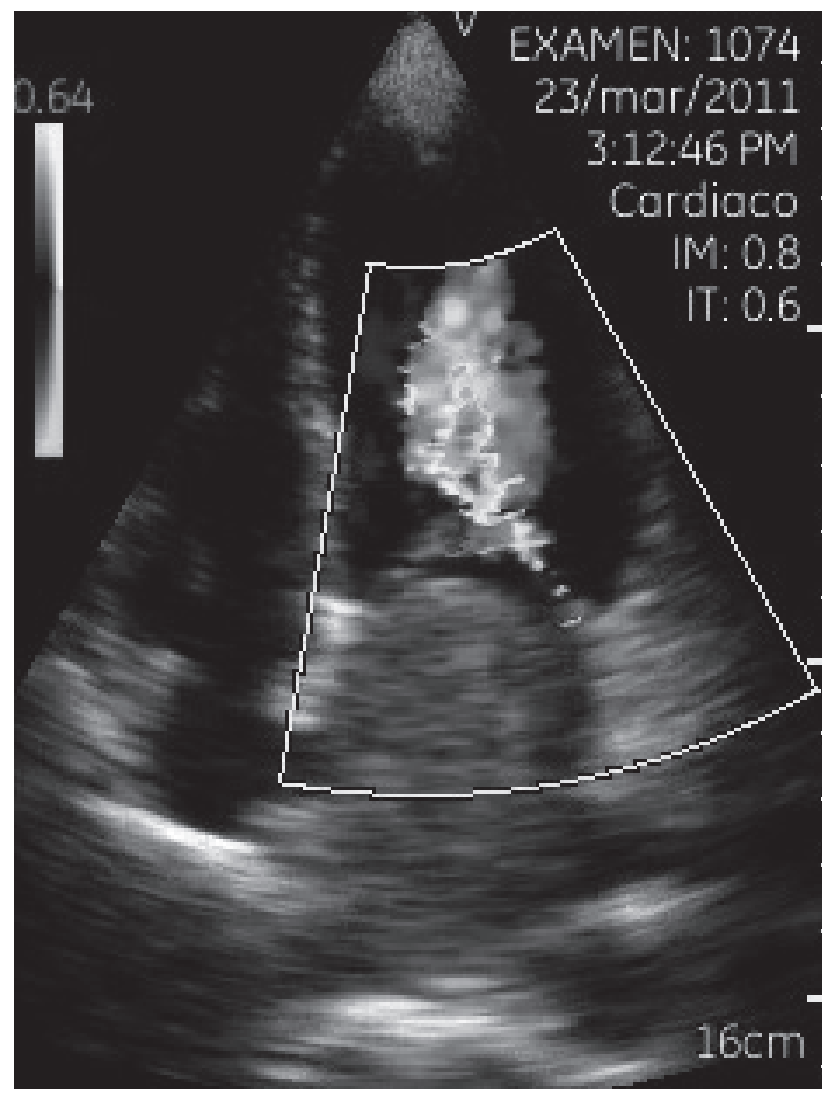


Se consideró un índice por encima del 80\% como muy bueno; del 41 al 80\% como intermedio; y menor o igual al 40\% como malo. Para variables continuas se usó la correlación de Spearman. Subdivisión de variables categóricas como: Leve, Moderada y Severa.

Figura 6. Correlación de la Fracción de Eyección ecógrafo de bolsillo/convencional

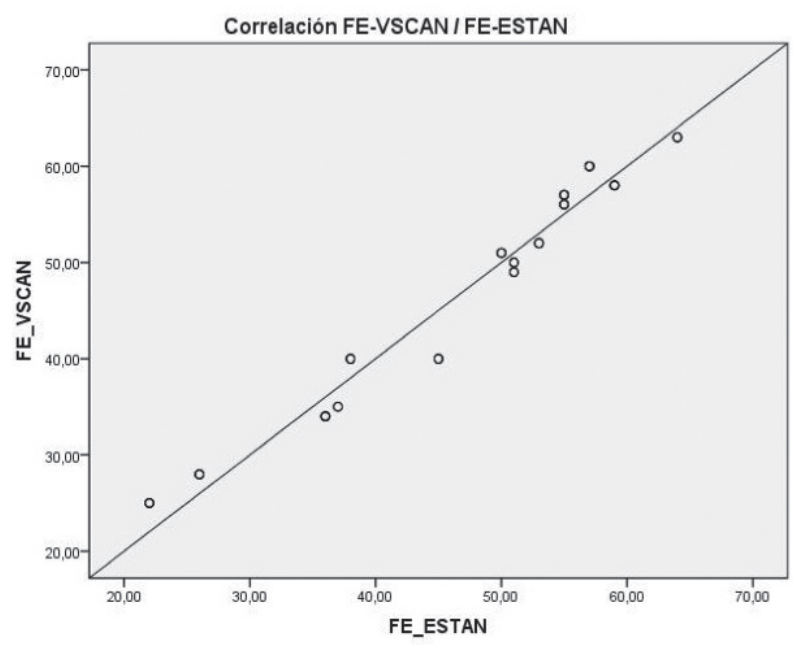

\section{Resultados}

Durante un periodo de 24 meses, se han realizado exploraciones con ecografía de bolsillo (GE VSCAN) a un total de 1.025 pacientes ingresados en nuestro hospital, dependientes del Servicio de Medicina Interna y otros Servicios Médicos y Quirúrgicos. En total se han archivado un total de 5.200 registros (secuencias de vídeo e imágenes estáticas), con una media de 5 registros por paciente. El tiempo medio empleado en la exploración ecocardiográfica básica fue de 5 minutos, la abdominal de 4 y ambas en menos de 10 minutos.

Se pudieron analizar y comparar los resultados de 821 ecocardiografías realizadas con el VSCAN y el ecocardiógrafo convencional (Tabla 1). EI VSCAN objetivó algún hallazgo patológico en 681(83\%) exploraciones (Figura 5). El nivel de concordancia fue muy bueno para las variables líquido pericárdico, fracción de eyección (Figura 6), hipertrofia del ventrículo izquierdo, vegetaciones, tumor o trombo intracardiaco, dilatación de cavidades, insuficiencia tricúspide e insuficiencia mitral. La concordancia fue intermedia para estenosis mitral, insuficiencia pulmonar y estenosis aórtica. La concordancia fue también excelente con las ecografías de tórax para el diagnóstico del derrame pleural.

Se realizaron y compararon los resultados de 810 ecografías abdominales que demostraron algún hallazgo patológico en el VSCAN en 688 (85\%) casos (Tabla 1). La concordancia con la ecografía convencional fue muy buena para ascitis, dilatación de la vena cava y de las suprahepáticas, esteatosis hepática, hepatoesplenomegalia, litiasis biliar, hidronefrosis, quistes renales, globo vesical y tamaño renal. La concordancia fue intermedia para las variables colecistitis, dilatación de la vía biliar, aneurisma aórtico abdominal, metástasis hepáticas, dilatación de la vena porta, nefrolitiasis y tamaño prostático.

Se realizaron 120 técnicas guiadas por VSCAN (ayuda en la localización del punto de punción): 60 toracocentesis, 48 paracentesis, 1 drenaje de absceso y una pericardiocentesis (en $\mathrm{UCI})$, todas con éxito y sin complicaciones. De forma complementaria se han realizado conjuntamente con el Servicio de Neurocirugía 10 ecografías intraoperatorias con objeto de valorar su utilidad en la hidrocefalia (ecografía transfontanelar) y en la localización de LOES a través de una craneotomía.

Tabla 1. Nivel de concordancia - correlación entre los hallazgos de la ecografía de bolsillo y la convencional

\begin{tabular}{|l|c|}
\hline \multicolumn{2}{|c|}{ Ecocardiografía y ecografía torácica } \\
\hline Líquido pericárdico & $(k=0,94, P<0,01)$ \\
\hline Fracción de eyección del VI & $(r=0.92, P<0,01)$ \\
\hline Hipertrofia de ventrículo izquierdo & $(k=0,85, P<0,01)$ \\
\hline Vegetaciones, tumor o trombo intracardiaco & $(k=0,88, P<0,01)$ \\
\hline Dilatación de cavidades & $(k=0,87, P<0,01)$ \\
\hline Insuficiencia tricúspide & $(k=0,85, P<0,01)$ \\
\hline Insuficiencia mitral & $(k=0,84, P<0,01)$ \\
\hline Estenosis mitral & $(k=0,75, P<0,01)$ \\
\hline Insuficiencia pulmonar & $(k=0,65, P<0,01)$ \\
\hline Estenosis aórtica & $(k=0,62, P<0,01)$ \\
\hline Derrame pleural & $(k=0,98, P<0,001)$ \\
\hline
\end{tabular}

\begin{tabular}{|l|c|}
\hline \multicolumn{2}{|c|}{ Ecografía abdominal } \\
\hline Ascitis & $(k=0,95, P<0,01)$ \\
\hline Dilatación de cava y suprahepáticas & $(k=0,85, P<0,001)$ \\
\hline Dilatación de la vena porta & $(k=0,68, P<0,01)$ \\
\hline Esteatosis hepática & $(k=0,85, P<0,01)$ \\
\hline Hepato-esplenomegalia & $(k=0,82, P<0,01)$ \\
\hline Metástasis hepáticas & $(k=0,53, P<0,01)$ \\
\hline Litiasis biliar & $(k=0,92, P<0,01)$ \\
\hline Dilatación vía biliar & $(k=0,74, P<0,001)$ \\
\hline Colecistitis & $(k=0,68, P<0,01)$ \\
\hline Hidronefrosis & $(k=0,95, P<0,01)$ \\
\hline Quistes renales & $(k=0,93, P<0,01)$ \\
\hline Globo vesical & $(k=0,93, P<0,01)$ \\
\hline Tamaño renal & $(r=0,82 P<0,01)$ \\
\hline Tamaño prostático & $(r=0,75, P<0,01)$ \\
\hline Nefrolitiasis & $(k=0,62, P<0,01)$ \\
\hline Aneurisma aórtico abdominal & $(k=0,72, P<0,001)$ \\
\hline
\end{tabular}




\section{Discusión}

La miniaturización de los dispositivos portátiles de ultrasonidos hasta adquirir un tamaño de bolsillo verdaderamente anuncian el estetoscopio ultrasónico ${ }^{1,12-14}$. La realización de ecocardiografías con dispositivos portátiles ha demostrado ser factible en diferentes entornos ${ }^{15,16}$, y estudios recientes muestran la exactitud de los hallazgos obtenidos con la ecocardiografía de bolsillo en diferentes parámetros clínicos y pacientes de diferentes poblaciones ${ }^{17,18}$. El uso de un dispositivo de ultrasonido de 'bolsillo' por un cardiólogo entrenado en ecocardiografía sabemos que puede estimar la función ventricular izquierda y fracción de eyección en más de tres cuartas partes de pacientes cardíacos que acuden a un centro hospitalario ${ }^{19}$. La utilidad clínica diagnóstica y los potenciales usos en la clínica práctica de estos dispositivos han sido motivo de diversos estudios y foco de atención de diversos organismos internacionales en ecocardiografía $^{14,20,21}$. Los estudios de Kobal ${ }^{22}$ mostraron la rentabilidad de la ecocardiografía básica utilizada por estudiantes de medicina con un mínimo de formación contrastado con los resultados de la exploración física realizada por cardiólogos expertos en pacientes con cardiopatía. La valoración básica ecocardiografíca de la función y morfología cardiaca y la interpretación de las imágenes obtenidas con un dispositivo de bolsillo cuando se realiza por ecocardiografistas experimentados muestra una correlación de moderada a muy buena a la obtenida con equipo estándar ${ }^{23,24}$. La valoración de hipertrofia de ventrículo izquierdo con un dispositivo de ecografía de bolsillo como método de screening ha sido motivo de investigación ${ }^{25}$. En nuestro estudio, con el manejo de esta técnica por internistas como un complemento a la exploración física, se obtiene un nivel de concordancia bueno 0 muy bueno en la mayoría de parámetros evaluados respecto a la ecografía convencional tras un proceso de aprendizaje del explorador. La ecografía de bolsillo fue especialmente útil en la valoración del derrame pericárdico, dilatación de cavidades cardiacas, hipertrofia de ventrículo izquierdo, estimación de la función y fracción de eyección de ventrículo izquierdo (visual), insuficiencia mitral y tricúspide. Nuestros resultados son similares a lo publicado por Kimuraet al ${ }^{26}$ en la valoración de la estimación de la función del ventrículo izquierdo y del tamaño de aurícula izquierda. La concordancia en la estimación de valvulopatía aórtica, y su cuantificación es ciertamente inferior. Hay que recordar que el dispositivo utilizado no dispone de Doppler pulsado, continuo, ni modo $\mathrm{M}$, por lo que en absoluto puede reemplazar un estudio cardiológico completo en el estudio profundo de estas valvulopatías. En cuanto a la valoración de abdomen conviene resaltar que el dispositivo dispone de una sonda sectorial, con un programa para abdomen, que no es la sonda más idónea para esta localización, donde se prefiere una sonda convexa. No obstante, y a pesar de esta limitación técnica, la exploración con el VSCAN es muy útil en la detección de líquido ascítico, globo vesical, signos de hidronefrosis o litiasis biliar y por tanto, a nuestro juicio, es muy útil en una primera valoración del paciente, en urgencias o consulta. Hemos obtenido una concordancia inferior en la detección de metástasis hepáticas, lo que su utilidad real en el despistaje tumoral es escasa. En todo caso la utilidad de la ecografía de bolsillo no es sustituir la realización de ecografías o TACs regladas sino añadir otros datos a los obtenidos en la historia y la exploración física de los pacientes, que permitan tomar decisiones diagnósticas o terapéuticas más correctas y en menor tiempo. Además conviene conocer las limitaciones del dispositivo que utilizamos, como la imposibilidad de intercambiar sondas, por lo que no permite la valoración del tiroides, la ayuda al acceso de vías venosas centrales ó la valoración de una posible trombosis profunda en los miembros inferiores, situaciones para las que se precisaría una sonda lineal, de la que carece el dispositivo. Sin duda en un futuro no lejano vamos a disponer de varios modelos de dispositivos de bolsillo con un precio contenido (el precio actual del VSCAN está alrededor de los $6.000 €$ ).

En una técnica tan ligada a la experiencia y habilidades del explorador como es la ecografía, probablemente los resultados mejorarán con la experiencia creciente de los exploradores y su conocimiento de las limitaciones del dispositivo. En nuestro estudio hemos apreciado una mejor concordancia en los resultados parciales de las exploraciones realizadas durante el segundo año en relación al primero. Nuestros resultados están también condicionados por el carácter no experimental del estudio, que sí está integrado en la atención clínica diaria a nuestros pacientes. Por tanto seguimos necesitando más trabajos, realizados por médicos expertos, que exploren la eficacia, validez y utilidad de esta nueva técnica.

La formación en ecografía básica empieza a incluirse actualmente en Estados Unidos (Consejo de Acreditación en Educación Médica) en los programas de residencia en Graduados de Medicina de Emergencia. Del mismo modo, es aconsejable que todos los nuevos Internistas reciban una formación adecuada en ecografía, a fin de conseguir al menos un primer nivel básico y que dicha formación sea acreditada y probada.

Así, tras un período de formación teórica y práctica breve, el internista podrá determinar la dimensión y función de ambos ventrículos, detectar la presencia de derrame pericárdico significativo, orientar el diagnóstico de valvulopatías severas ó estimar la presión venosa central mediante el diámetro y colapsabilidad de la vena cava inferior con elevada fiabilidad, entre otras habilidades ${ }^{27}$. La curva de aprendizaje 
de la ecografía básica difiere entre los diferentes trabajos publicados en la literatura, pero oscila entre las 6 y las 16 horas e incluye teoría y práctica supervisada por un profesor experto $^{27}$ y al menos entre 20 y 40 estudios supervisados posteriormente ${ }^{28}$. La formación continuada y la realización de cursos generales y específicos son imprescindibles posteriormente. La inclusión de la práctica de la ecografía en el mapa de competencias básicas de la Medicina Interna es un acierto pues en un futuro cercano su manejo será imprescindible para cualquier internista ${ }^{29,30}$. La incorporación de un ecógrafo de bolsillo creemos supera la rentabilidad diagnóstica respecto a la exploración clínica de rutina y mejora el manejo terapéutico, disminuyendo sin duda los días de estancia hospitalaria al disminuir la lista de espera de pruebas complementarias con el consiguiente ahorro económico. La realización de una ecografía de rutina a los pacientes ingresados en Medicina Interna puede cambiar de manera significativa el diagnóstico y manejo obtenido en un $20 \%$ de los $\operatorname{casos}^{31}$.

Animamos a todos los internistas a integrarse y formar parte del grupo de trabajo de Ecografía Clínica de la SEMI, visitar la página web (www.ecografiaclinica.es) donde se informa de las actividades del grupo, manuales, casos clínicos, formación, cursos y su participación en el grupo de Facebook.

\section{Conclusión}

La ecografía de bolsillo es un instrumento útil en la práctica diaria del internista. Permite obtener información valiosa para la toma de decisiones, más allá de la exploración física, a pie de cama del paciente. Además es particularmente útil como ayuda en la realización de algunas técnicas muy habituales en la clínica, como toracocentesis y paracentesis. La calidad de imagen obtenida con la ecografía de bolsillo con doppler color es buena y suficiente para realizar una primera valoración ecográfica que puede adelantar un diagnóstico y no demorar un tratamiento.

\section{Conflicto de intereses}

Ninguno.

\section{Bibliografía}

1. Popp RL: The physical examination of the future: echocardiography as part of the assessment. ACC Curr Rev 1988;XX:79-81.

2. Liebo MJ, Israel RL, Lillie EO, et al. Is pocket mobile echocardiography the nextgeneration stethoscope? A cross-sectional comparison of rapidly acquired images with standard transthoracic echocardiography. Ann Intern Med 2011; 155: 33-8 .

3. Marwick TH. The future of echocardiography. Eur J Echocardiogr 2009; 10: 594-601.

4. Choi BG, Mukherjie M, Dala P, et al. Interpretation of remotely downloaded pocketsize cardiac ultrasound images on a web-enabled smartphone. J Am Soc Echocardiogr 2011; 24: 1325-30.

5. Alexander $\mathrm{JH}$, Peterson PD, Chen AY, et al. Feasibility of point of care echo by noncardiologist physicians to assess left ventricular function, pericardial effusion, mitral regurgitation, and aortic valve thickening. Circulation 2001; 104(Suppl. II): 334.

6. Beaulieu Y, Marik PE. Bedside ultrasonography in the ICU (Part 1). Chest 2005; 128 : 881-95.

7. Vignon P, Dugard A, Abraham J, et al. Focused training for goal-oriented hand-held echocardiography performed by non-cardiologist residents in the intensive care unit. Intensive Care Med 2007; 33: 1795-9.

8. Reardon R, Heegaard B, Plummer D, Clinton J, Cook T, Tayal V. American College of Emergency Physicians (2009) Emergency ultrasound guidelines. Ann Emerg Med 2009; 53: 550-70.

9. Arienti V, Camaggi, V. Clinical applications of bedside ultrasonography in internal and emergency medicine. Intern Emerg Med 2011; 6: 195-201.

10. Andersen GN, Hangen BO, Graven T, et al. Feasibility and reliability of point-of-care pocket-sized echocardiog- raph. Eur J Echocardiogr 2011; 12: 665-70 . 11.

12. Roelandt JR. A personal ultrasound imager (ultrasound stethoscope): a revolution in the physical cardiac diagnosis. Eur Heart J 2002; 23: 523-7.

13. Kimura BJ, DeMaria AN. Hand-carried ultrasound: evolution, not revolution. Nat Clin Pract Cardiovasc Med 2005; 4:217-23.

14. Prinz C, Voigt JU. Diagnostic accuracy of a hand-held ultrasound scanner in routine patients referred for echocar- diography. J Am Soc Echocardiogr 2011; 24: 111-6 .

15. Vourvouri EC, Poldermans D, de Sutter J, et al. Experience with an ultrasound stethoscope. J Am Soc Echocardiogr 2012; 15: 80-5.

16. Bruce CJ, Montgomery SC, Bailey KR, et al. Utility of hand- carried ultrasound devices used by cardiologists with and without significant echocardiographic experience in the cardiology inpatient and outpatient settings . Am J Cardiol 2002; 90: 1273-5.

17. Culp BC, Mock JD, Chiles CD, et al. The pocket echocardiograph: Validation and feasibility. Echocardiography 2010; 27: 759-64.

18. Culp BC, Mock JD, Ball TR, et al. The pocket echocardiograph: A pilot study of its validation and feasibility in intubated patients. Echocardiography 2011; 28: 371-7.

19. Egan M, Ionescu A. The pocket echocardiograph: a useful new tool?. Eur J Echocardiogr. 2008; 9:721-5.

20. Sicari R, Galderisi M, Voigt JU, et al. The use of pocket- size imaging devices: A position statement of the European Association of Echocardiography. Eur J Echocardiogr 2011; 12: 85-7.

21. Seward JB, Douglas PS, Erbel R, et al. Hand-carried cardiac ultrasound (HCU) device: Recommendations regard- ing new technology. A Report from the Echocardiography Task Force on New Technology of the Nomenclature and Standards Committee of the American Society of Echocardiography. J Am Soc Echocardiogr 2002; 15: 369-73.

22. Kobal SL, Trento L, Baharami S, et al. Comparison of effectiveness of hand-carried ultrasound to bedside cardiovascular physical examination. Am J Cardiol 2005; 96 : 1002-6.

23. Prinz C, Dohrmann J, van Buuren F, et al. Diagnostic Performance of Handheld Echocardiography. Echocardiography 2012; 29: 887-94.

24. Fukuda S, Shimada K, Kawasaki T, et al. Pocket-sized transthoracic echocardiography device for the measurement of cardiac chamber size and function. Circ J 2009; 73: 1092-6.

25. Senior R, Galasko G, Hickman M, et al. Community screening for left ventricular hypertrophy in patients with hypertension using hand-held echocardiography . J Am Soc Echocardiogr 2004; 17: 56-61

26. Bruce J, Kimura MD, Glynn W, et al. Diagnostic performance of a pocket-sized ultrasound device for quick-look cardiac imaging. American Journal of Emergency Medicine 2012; 30: 32-6.

27. Torres Macho, J, Garcia de Casasola, G, Conthe Gutiérrez, P. Ecocardiografía clínica básica en Medicina Interna. Revista Clínica Española 2012; 212:141-6.

28. Vignon P, Mucke F, Bellec F, et al. Basic critical care echocardiography: validation of a curriculum dedicated to noncardiologist residents. Crit Care Med 2011; 39: 636-42.

29. Porcel JM, Casademont J, Conthe P, Pinilla B, Pujol R, García-Alegría J. Competencias básicas de la Medicina Interna. Rev Clin Esp 2011; 211: 307-11.

30. Formiga F. El ecocardiograma en la insuficiencia cardíaca, de la teoría a la práctica. Rev Clin Esp 2011; 211: 352-3.

31. Mjolstad OC, Dalen H, Graven T, et al. Routinely adding ultrasound examinations by pocket-sized ultrasound devices improves inpatient diagnostics in a medical department. Eur J Intern Med 2012; 23:185-91. 